BNL-96819-2012-IR

$\mathrm{C}-\mathrm{A} / \mathrm{AP} / \# 444$

Jan. 2012

\title{
High intensity protons in RHIC
}

C. Montag, L. Ahrens, M. Blaskiewicz, J. M. Brennan, K. A. Drees, W. Fischer, H. Huang, M. Minty, G. Robert-Demolaize, P. Thieberger, K. Yip

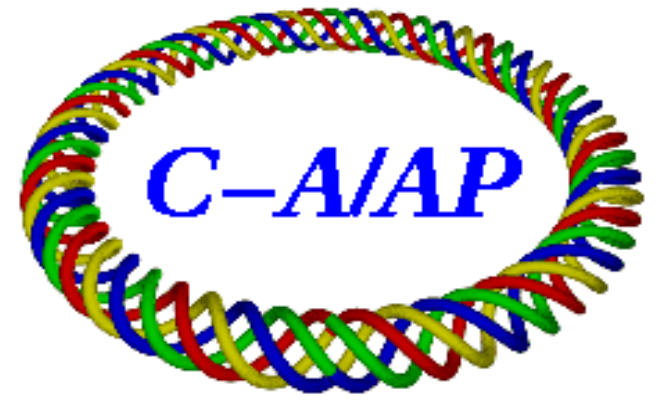

Collider-Accelerator Department Brookhaven National Laboratory Upton, NY 11973

Notice: This document has been authorized by employees of Brookhaven Science Associates, LLC under Contract No. DE-AC02-98CH10886 with the U.S. Department of En ergy. The United States Government retains a nonexclusive, paid-up, irrevocable, world-wide license to publish or reproduce the published form of this document, or allow others to do so, for United States Government purposes. 


\title{
High Intensity Protons in RHIC
}

\author{
C. Montag, L. Ahrens, M. Blaskiewicz, J. M. Brennan, K. A. Drees, W. Fischer, \\ H. Huang, M. Minty, G. Robert-Demolaize, P. Thieberger, K. Yip
}

January 5, 2012

\begin{abstract}
During the 2012 summer shutdown a pair of electron lenses will be installed in RHIC, allowing the beam-beam parameter to be increased by roughly 50 percent. To realize the corresponding luminosity increase bunch intensities have to be increased by 50 percent, to $2.5 \cdot 10^{11}$ protons per bunch. We list the various RHIC subsystems that are most affected by this increase, and propose beam studies to ensure their readiness.
\end{abstract}

\section{Introduction}

The proton luminosity in RHIC is presently limited by the beam-beam effect. To overcome this limitation, electron lenses will be installed in IR10. With the help of these devices, the headon beam-beam kick experienced during proton-proton collisions will be partially compensated, allowing for a larger beam-beam tuneshift at these collision points, and therefore increasing the luminosity. This will be accomplished by increasing the proton bunch intensity from the presently achieved $1.65 \cdot 10^{11}$ protons per bunch in 109 bunches per beam to $2.5 \cdot 10^{11}$, thus roughly doubling the luminosity. In a further upgrade we aim for bunch intensities up to $3 \cdot 10^{11}$ protons per bunch.

With RHIC originally being designed for a bunch intensity of $1 \cdot 10^{11}$ protons per bunch in 56 bunches, this six-fold increase in the total beam intensity by far exceeds the design parameters of the machine, and therefore potentially of its subsystems. In this note, we present a list of major subsystems that are of potential concern regarding this intensity upgrade, show their demonstrated performance at present intensities, and propose measures and beam experiments to study their readiness for the projected future intensities.

\section{Ramp Efficiency}

During FY-11 bunch intensities up to $2 \cdot 10^{11}$ protons/bunch in 109 bunches per ring were successfully ramped to $250 \mathrm{GeV}$, with ramp efficiencies of 95 percent (Figure 1). Losses on the ramp occured predominantly during the final squeeze due to the larger emittance associated with higher intensity bunches delivered by the AGS. With the new polarized proton source, which is expected to be capable of delivering ten times higher intensities than the present one, more aggressive scraping can be applied in the Booster, which is expected to result in smaller emittances at AGS extraction. These smaller emittances will in turn lead to improved ramp efficiencies at high intensity.

\section{Polarization Transmission}

The polarization transmission efficiency on the RHIC ramp from injection to $250 \mathrm{GeV}$, as defined by

$$
\eta=\frac{P_{250 \mathrm{GeV} \text { store }}}{P_{\text {injection }}}
$$



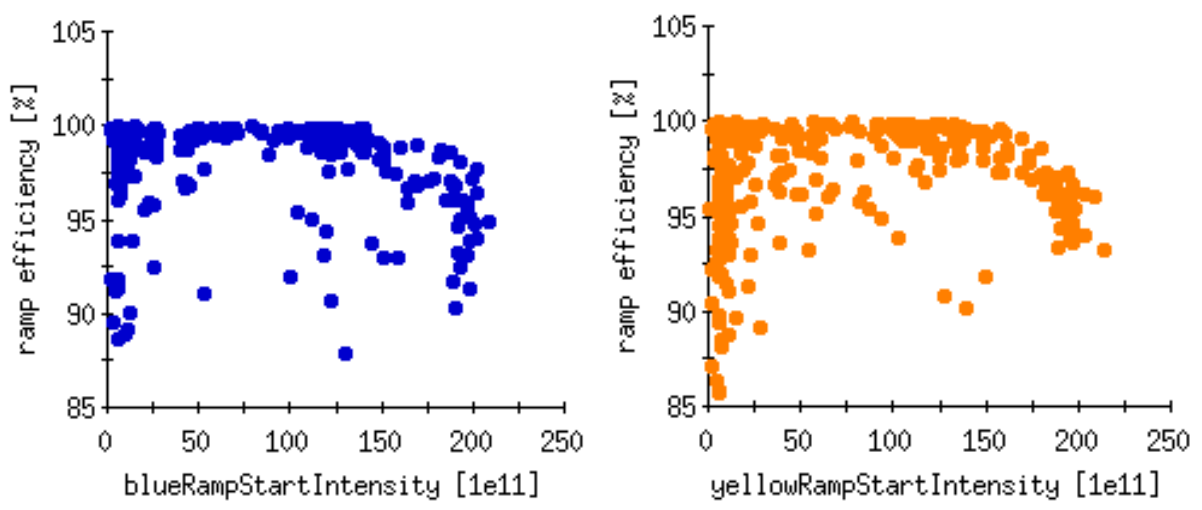

Figure 1: RHIC polarized proton ramp efficiencies in the Blue (left) and Yellow (right) ring during the FY-11 polarized proton run at $250 \mathrm{GeV}$.

did not exhibit any intensity dependence during FY-11 (Figure 2). However, higher intensity bunches had lower polarization at AGS extraction, and therefore at RHIC injection, than lower intensity ones. It is believed that this results from higher intensity bunches having larger transverse emittances in the AGS. Since particles at large vertical amplitudes experience stronger horizontal magnetic fields in the AGS quadrupoles, their polarization tends to be lower. With the new polarized proton source, emittances in the AGS are expected to be smaller, thus resulting in higher polarization.

\section{Radiation Safety}

The Accelerator Safety Envelope (ASE) has recently been increased to $5 \cdot 10^{13}$ protons per beam, or $4.5 \cdot 10^{11}$ protons per bunch in 109 bunches at $250 \mathrm{GeV}$ [1]. However, for FY-12 RHIC will still be limited to half that intensity, since new fences around ventilation shafts as well as improved shielding walls will not be installed before next summer. Once these upgrades are in place, they need to be reviewed before RHIC can actually operate at these higher intensities. However, for short commissioning tests temporary precautions can be put in place, reviewed, and approved.

The radiation dose, except for muons, scales as $E^{0.8}$. Therefore, at a lower beam energy of $100 \mathrm{GeV}$, much higher beam intensities are permissible.

\section{$5 \quad$ Scrubbing}

To reduce the electron cloud induced pressure rise, beam pipe scrubbing is necessary. Bunches at AGS extraction will be shortened by quad mode pumping, and captured with the $9 \mathrm{MHz} \mathrm{RF}$ system in RHIC. In RHIC, bunches will be shortened further by rebucketing into the $197 \mathrm{MHz}$ storage RF system.

When scrubbing was last attempted in FY-09, radiation losses in the Yellow abort kicker area caused false fire alarms, which prevented effective scrubbing. In preparation for the scrubbing effort in FY-12, the RHIC fire alarm system in IR10 has been inspected during a walk-through of that area. Since this particular area is equipped with sprinklers that are triggered by heat rather than smoke, the smoke detectors in IR10 will be temporarily disabled during scrubbing to avoid false alarms due to the increased radiation levels. The pretection provided by the sprinkler system was deemed sufficient by the BNL fire protection engineer and the fire captain. 


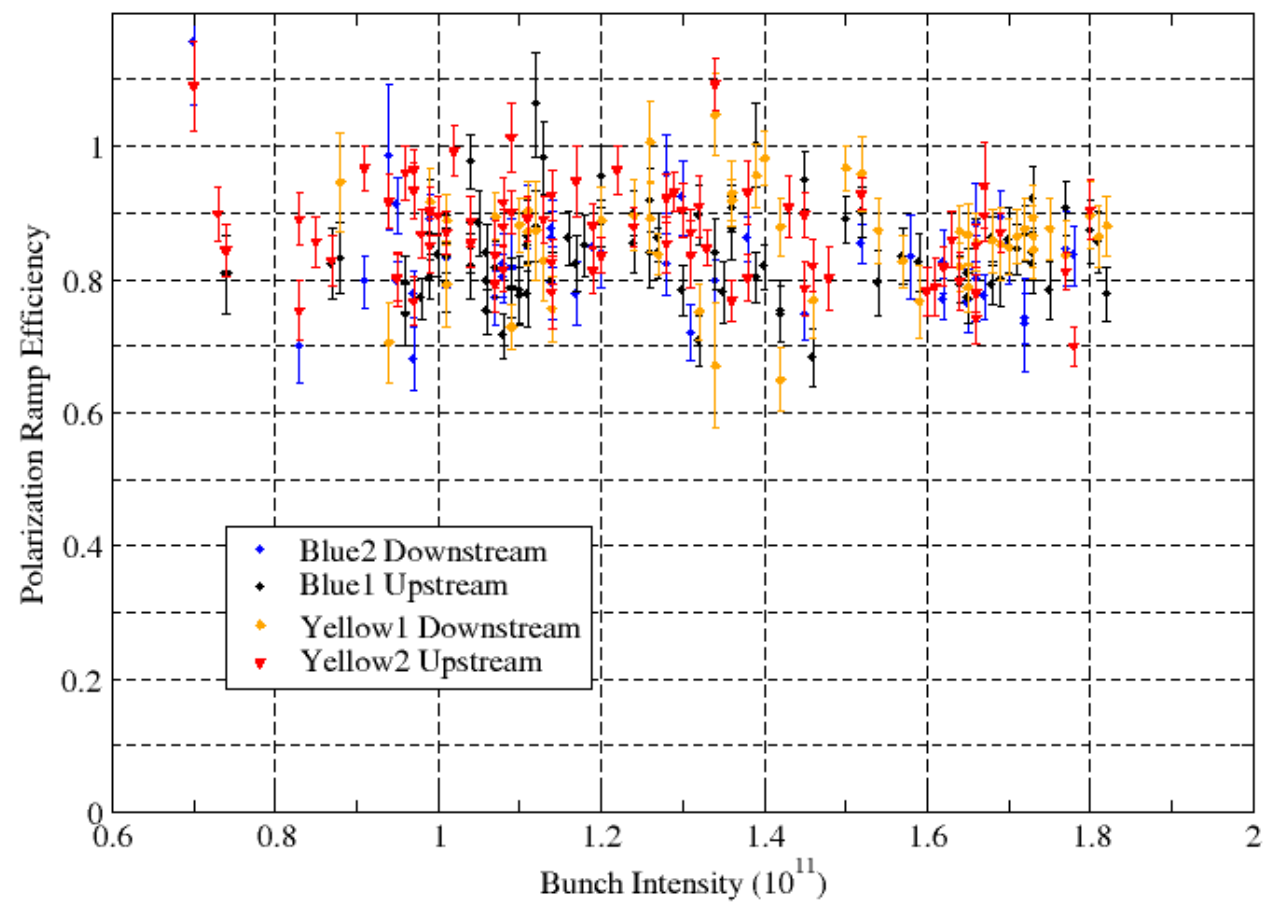

Figure 2: Polarization transmission $\eta=\frac{P_{250 \mathrm{GeV} \text { store }}}{P_{\text {injection }}}$ from injection to $250 \mathrm{GeV}$ during FY-11, as function of bunch intensity. 


\section{RHIC Beam Dump}

The RHIC beam dump was originally designed for a bunch intensity of $1 \cdot 10^{11}$ protons per bunch in 56 bunches per ring at $250 \mathrm{GeV}$, with a safety factor of 2 . This design was performed with three objectives:

- Maintain the integrity of the vacuum window through which the dumped beam must pass;

- maintain the integrity of the steel that absorbs most of the beam energy;

- protect the downstream superconducting magnets from quenching as a result of a beam dump.

When RHIC was operating with a bunch intensity of $1.2 \cdot 10^{11}$ protons per bunch in 109 bunches per ring, unintentional beam aborts early in the store, when the intensity is high, resulted in quenches of the first superconducting RHIC magnets downstream of the beam dump. Since at these intensities and bunch numbers the aforementioned safety factor was exceeded, this was not entirely unexpected.

To improve the situation MCNPX simulations were performed in order to design an upgrade to the system [2]. As it turned out, increasing the wall thickness of the beam pipe for the circulating RHIC beam next to the beam dump showed promising results in these simulations. Since thre were concerns about exposing the activated carbon in the beam dumps to air while replacing the beam pipe, the wall thickness of the existing pipe was increased by inserting twenty 5 inch long sleeves into the existing pipe. Figure 3 depicts one of these sleeves before installation. According to the MCNPX simulations, this upgraded RHIC abort system should now be capable of safely dumping proton bunch intensities up tp $2.5 \cdot 10^{11}$ protons per bunch in 109 bunches at $250 \mathrm{GeV}$.

After this modification, when RHIC was operating at bunch intensities up to $2 \cdot 10^{11}$ protons per bunch in 109 bunches at $250 \mathrm{GeV}$ during FY-11, no more beam induced magnet quenches were observed, thus verifying the validity of the MCNPX simulations. However, it is still unclear whether bunch intensities as high as $3 \cdot 10^{11}$ would again result in magnet quenches. It is therefore planned to study this during the FY-12 proton run, using the unpolarized high-intensity proton source. Since the MCNPX simulations showed good predictive power in upgrading the system, it is reasonable to assume that a further upgrade can be devised based on these simulations, if necessary. Beam loss monitors will be installed along the beam pipe in locations that, according to the MCNPX simulations, are representative of the beam losses that lead to magnet quenches. During these tests, the dependence of these losses on the abort kicker strength will be studied.

Another area of concern at high intensities is the vacuum window at the dump, and the dump itself. The localized energy deposition during a beam abort causes thermal stress in both the window and the steel of the dump itself, which may lead to cracks and therefore, in the case of the window, to vacuum failure. Simulation studies are currently being performed to investigate these issues in detail [3].

\section{Collimation System}

The present RHIC collimation system consists of one L-shaped double-plane jaw per ring acting as primary collimator and a series of three single-plane jaws installed directly downstream, acting as secondary collimators [4]. Figure 4 shows a recently inspected Yellow primary collimator. In the past, this system has been able to sufficiently reduce detector backgrounds for bunch intensities up to $2 \cdot 10^{11}$ protons per bunch in 109 bunches. Based on this experience, and assuming that backgrounds scale linearly with intensity, both STAR and PHENIX do not expect any problems with background at $3 \cdot 10^{11}$ protons per bunch. However, an upgraded system with newly located secondary collimators is being investigated as a back-up. To reduce the sensitivity to orbit changes, double sided secondary collimators have been proposed as part of this upgrade. 


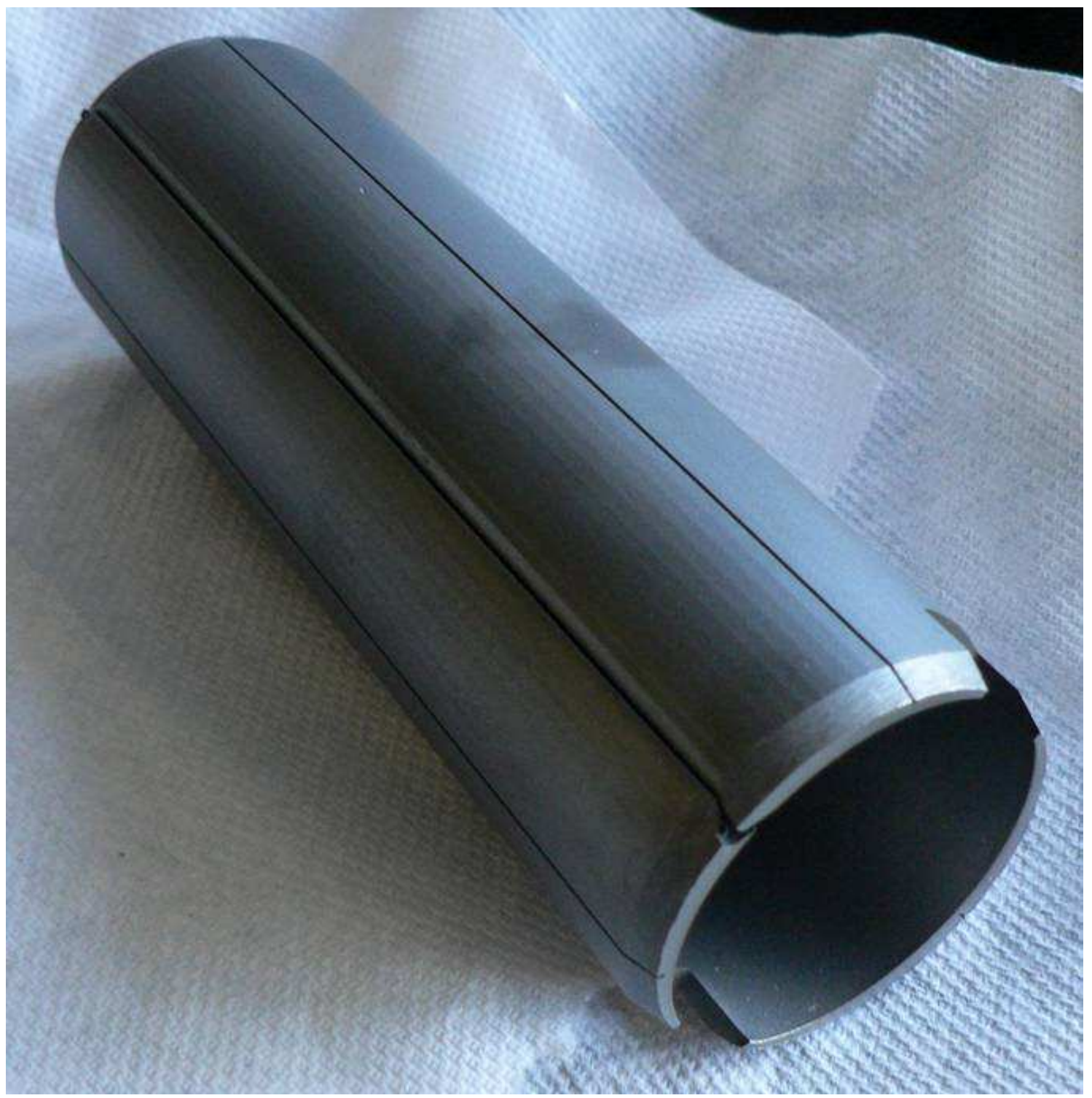

Figure 3: One of the sleeves inserted in the RHIC beam pipe adjacent to the dump to increase its wall thickness. 


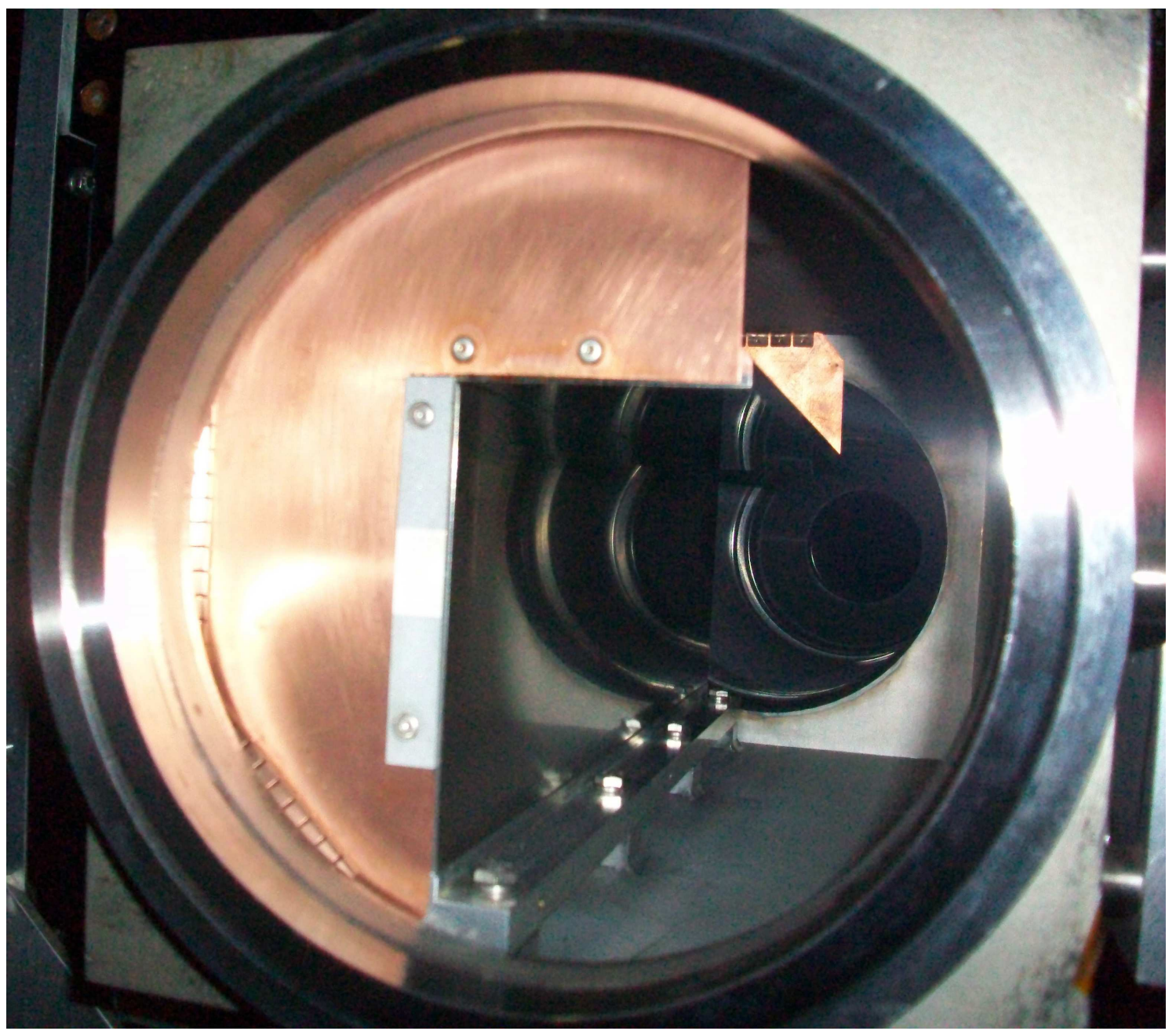

Figure 4: Recently inspected Yellow primary collimator, showing a flat surface without any damage. 
To compare the effect of the present and the proposed collimation system, a simulation code has been developed that tracks particles hitting the primary collimators to study the loss pattern around the circumference of the machine, as shown in Figures 5 and 6 [5]. The proposed new secondary collimators, which would be installed one arc downstream of the primary ones, would significantly reduce the loss rates in all arcs downstream of these secondaries, while the losses in the arc between primary and secondary collimators would remain essentially unchanged. Since this loss rate reduction is also true for the Blue triplet upstream of the STAR detector, the background situation at STAR is expected to improve significantly. In the Yellow ring, the new secodary collimators would be installed upstream of the STAR detector. According to these simulations, this also reduces the losses in the adjacent triplet just upstream of STAR. However, these simulations do not model the effect of showers generated at the collimators, which in this case might increase the STAR detector background from the Yellow beam. This effect therefore has to be investigated in further simulations.

\section{RF System}

The RHIC RF system consists of three different systems that serve different purposes during the RHIC ramp and store. At injection, the proton beam is captured in the $9 \mathrm{MHz}$ bucket, which is matched to the longitudinal phase space distribution provided by the AGS. An additional advantage of this system is the long bunch length during the ramp, which results in lower peak currents. Using this $9 \mathrm{MHz}$ system, the beam is then ramped to storage energy, where it is rebucketed twice, first into the $28 \mathrm{MHz}$ system and then into the $197 \mathrm{MHz}$ system.

The $9 \mathrm{MHz}$ system, schematically depicted in Figure 7, is fairly new and is therefore still being modified to maximize its performance. For Run-12, the cavity has been re-designed to lower its impedance by reducing its quality factor $Q$ from 2400 to 642 . Due to this modification, the $9 \mathrm{MHz}$ power amplifier is now better matched to this cavity. Additionally, a spark gap protection allows higher feedback gains to fight instabilities. This fast feedback reduces the cavity $R / Q=34.5 \Omega$ by a factor 30. The resulting shunt impedance is therefore $Z=Q \cdot(R / Q)=(34.5 \cdot 642 / 30) \Omega=738 \Omega$. Together with a beam curent of $I=0.9 \mathrm{~A}$, wich corresponds to $3 \cdot 10^{11}$ protons per bunch in 109 bunches, the cavity induced voltage per beam is computed as $U_{\text {ind }}=0.9 \mathrm{rmA} \cdot 738 \Omega=664 \mathrm{~V}$. Since this induced voltage is small compared to the cavity voltage of $20 \mathrm{kV}$, beam loading effects in the $9 \mathrm{MHz} \mathrm{RF}$ system are negligible.

The $28 \mathrm{MHz}$ system is not involved during injection and ramping. An offset frequency technique has been developed such that the cavity is always active on the ramp, but does not couple to the beam [6]. Due to this technique, the mechanical tuners do not have to move when the cavity is finally coupled to the beam for rebucketing at storage energy. The fundamental beam loading limit of the $28 \mathrm{MHz}$ system is expected to be around $9 \cdot 10^{11}$ protons per bunch in 109 bunches. However, dedicated beam studies are required to determine transient beam loading effects as well as higher order mode driven instabilities. These studies, which involve injecting, ramping, and rebucketing high intensity beams, will be carried out at $100 \mathrm{GeV}$ to avoid exceeding the ASE limits. Since the beam is stiffer at $250 \mathrm{GeV}$, the synchrotron frequency for a given voltage will be slightly higher at $100 \mathrm{GeV}$ than at $250 \mathrm{GeV}$. For a given voltage, the ratio of beam current to generator current does not change between 100 and $250 \mathrm{GeV}$. We can therefore be confident that results obtained at $100 \mathrm{GeV}$ are applicable at $250 \mathrm{GeV}$ as well.

The $197 \mathrm{MHz}$ system was originally common to both beams. In this configuration, power dissipation in the couplers limited the maximum bunch intensity to $1.3 \cdot 10^{11}$ protons per bunch in 109 bunches. To increase this limit, these common cavities have been separated such that they are no longer common to both beams, but dedicated to a single beam each. This increases the acceptable bunch intensity to at least $2.6 \cdot 10^{11}$ protons per bunch in 109 bunches. The actual limit will have to be determined in APEX studies. 

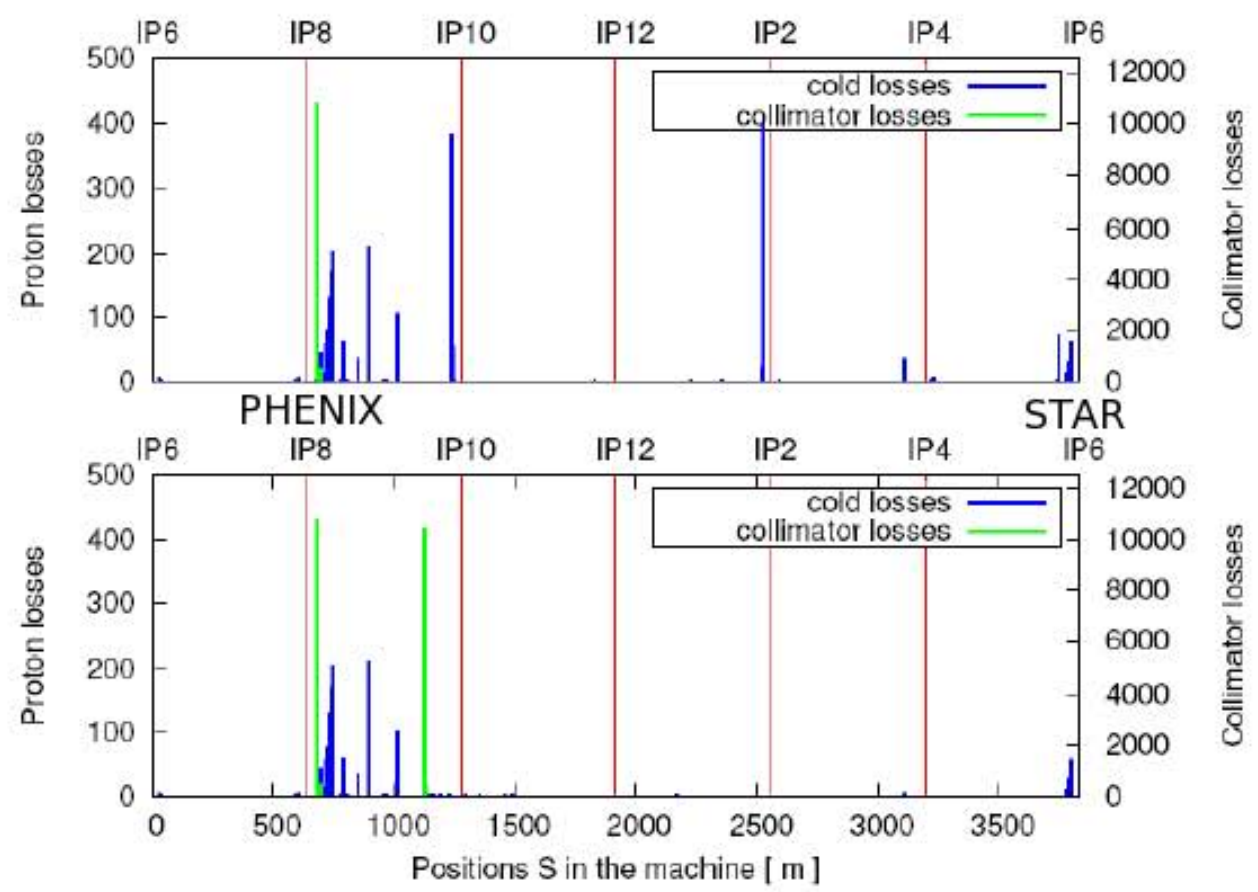

Comparison of longitudinal loss patterns between the current RHIC collimation system (top) and its proposed upgrade (bottom) for Blue Horizontal.
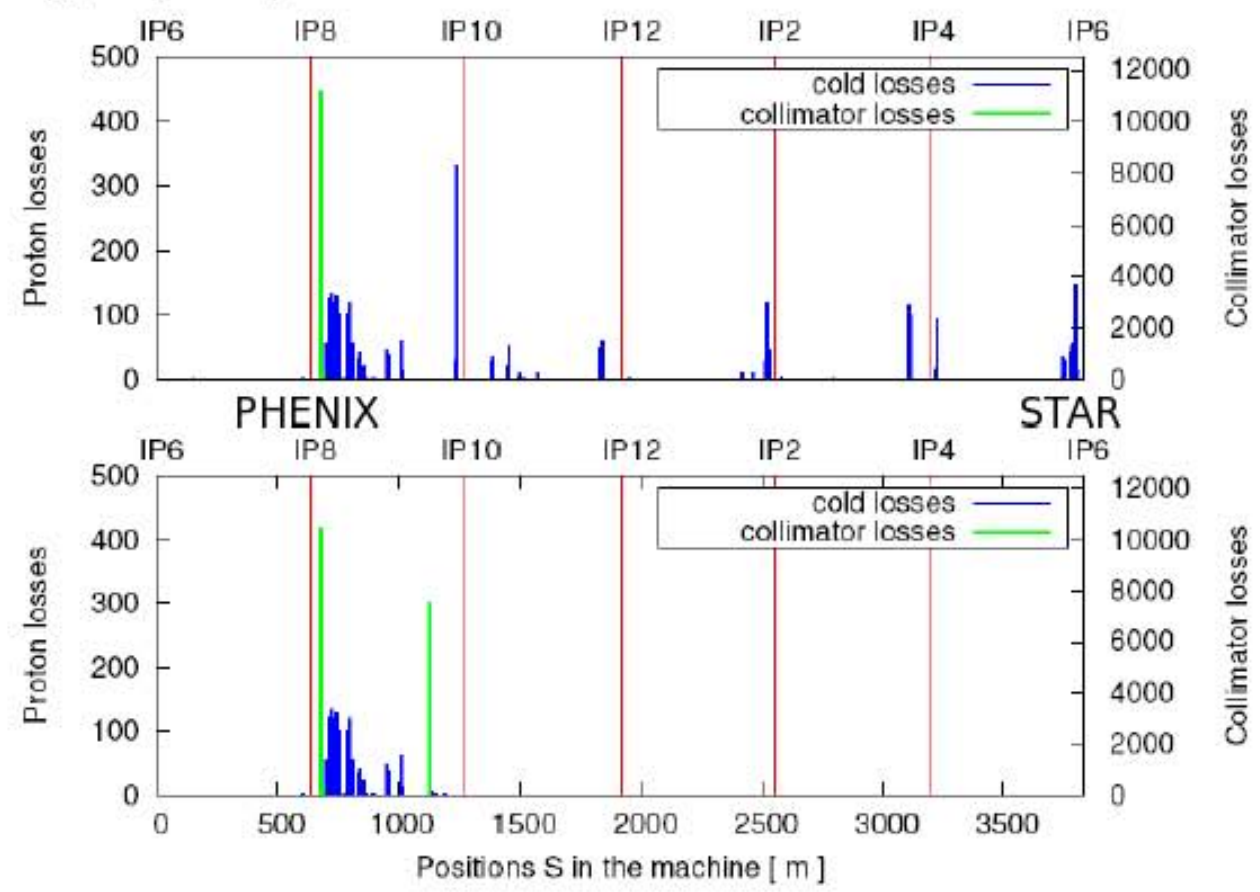

Comparison of longitudinal loss patterns between the current RHIC collimation system (top) and its proposed upgrade (bottom) for Blue Vertical.

Figure 5: Loss patterns for the Blue collimators. The beam direction is left-to-right. 

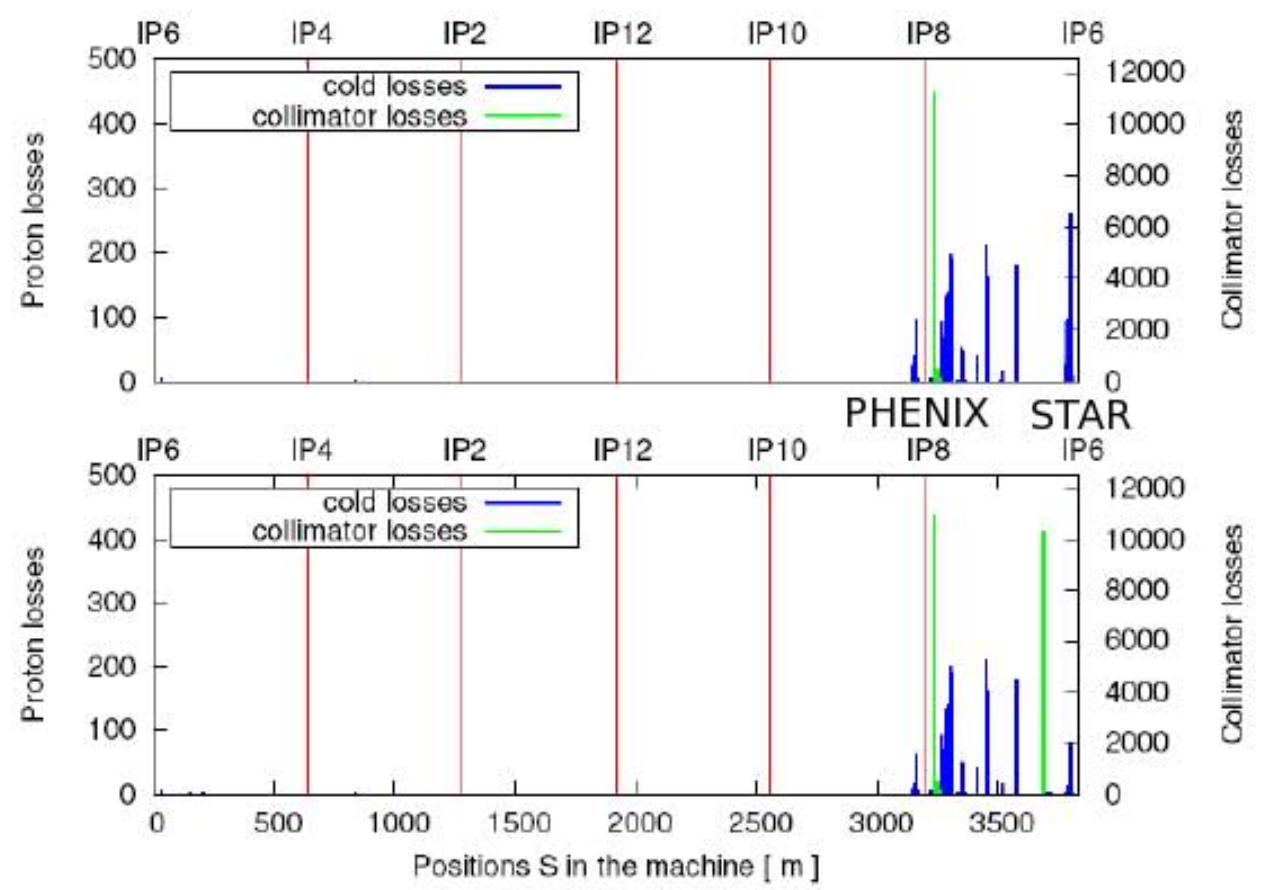

Comparison of longitudinal loss patterns between the current RHIC collimation system (top) and its proposed upgrade (bottom) for Yellow Horizontal.
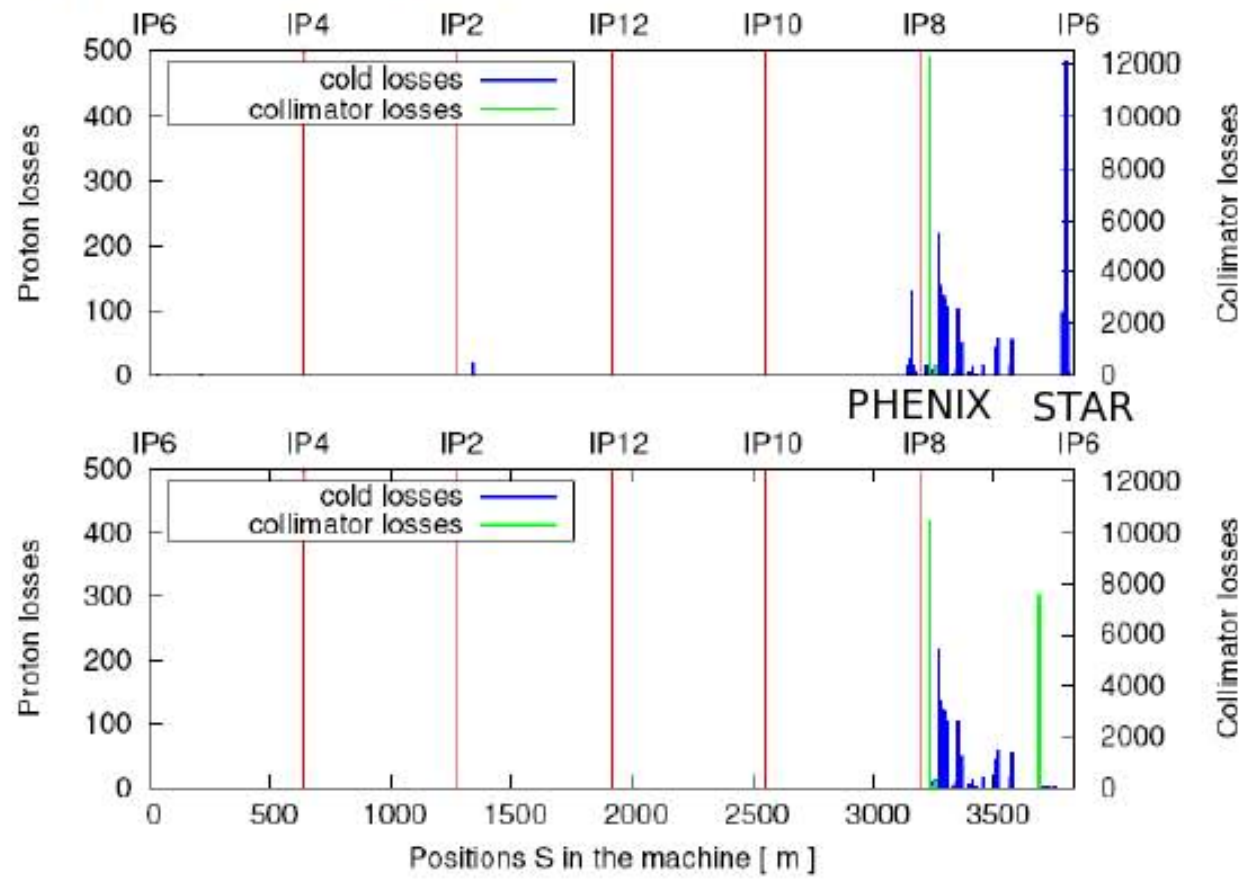

Comparison of longitudinal loss patterns between the current RHIC collimation system (top) and its proposed upgrade (bottom) for Yellow Vertical.

Figure 6: Loss patterns for the Yellow collimators. The beam direction is left-to-right. 


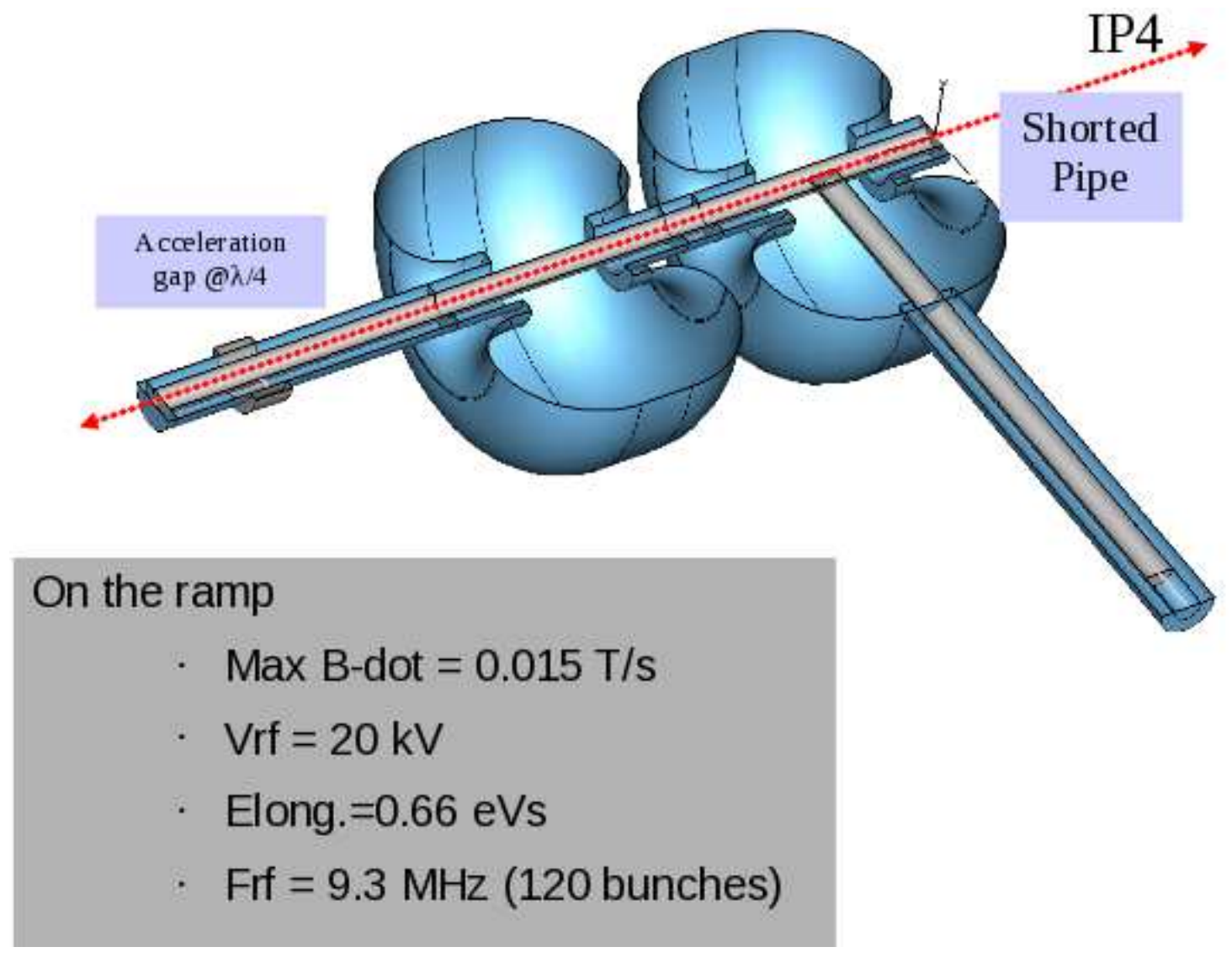

Figure 7: Schematic view of the $9 \mathrm{MHz}$ RF system in RHIC. 


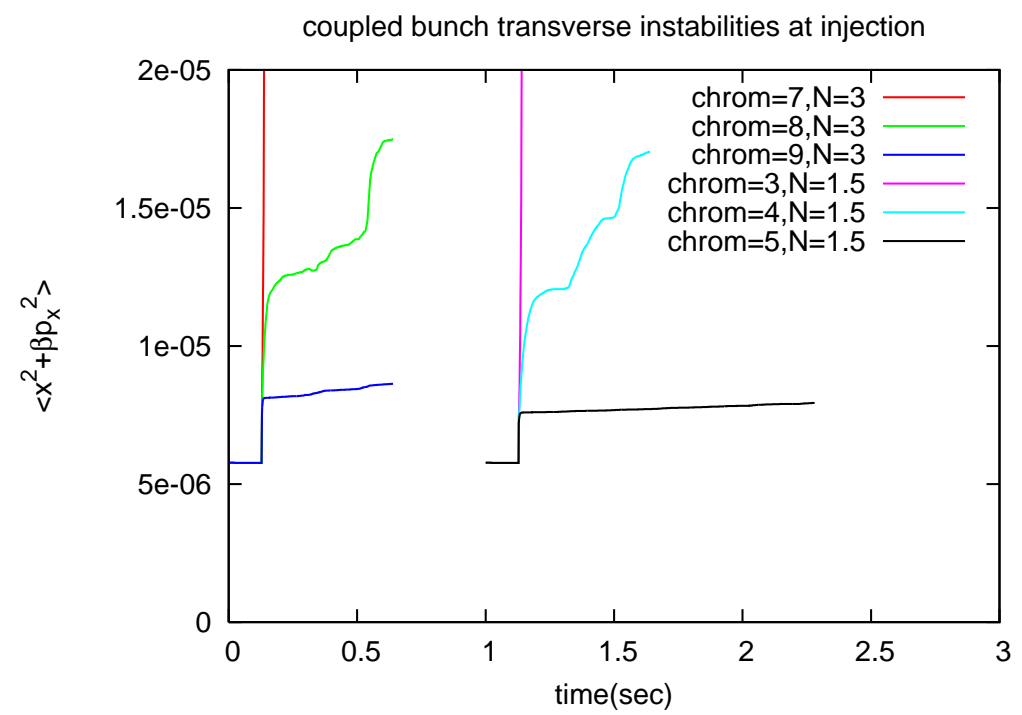

Figure 8: Simulation results for coherent instabilities at injection.

\section{Transverse Impedance}

The transverse impedance in RHIC was measured in 2002 at $Z_{\perp}=3-5 M \Omega$ [7]. Later measurements in 2010 showed a transverse impedance of $Z_{\perp}=18 M \Omega$ [8].

In the upcoming run, these measurements will be repeated, using the BBQ system as a highprecision tune meter. Bunches of different intensity will be injected into RHIC, and the tune of each individual bunch will be determined by gating the excitation kicker accordingly. The required accuracy of these tune measurements is estimated at a few $10^{-4}$, which is well within the capabilities of the BBQ system. Since $\Delta Q \propto Z_{\perp} /\left(\sigma_{z} E\right)$, short bunches at injection energy give the best resolution.

\section{Instabilities}

With proton bunch intensities as high as $2 \cdot 10^{11}$ protons per bunch in 109 bunches, RHIC never experienced any coherent instabilities that were not easily overcome by chromaticity corrections. To study the effect of higher bunch intensities, TRANFT simulations were performed [9]. These simulations include transverse impedances from direct and image current space charge effects, resistive wall, and the known impedances of BPMs, abort kickers and unshielded bellows. The longitudinal impedance is modeled as a broadband impedance chosen such that it yields the correct value for $|Z / n|$ [10]. Furthermore, injection beam parameters are chosen for these simulations, since they are expected to be most sensitive to coherent instabilites due to the low rigidity of the beam and the small synchrotron frequency of only $5 \mathrm{~Hz}$.

As Figure 8 indicates, 9 units of chromaticity would stabilize the beam at a bunch intensity of $3 \cdot 10^{11}$ protons per bunch. At presently typical bunch intensities of $1.5 \cdot 10^{11}$ protons per bunch, these simulations predict that 5 units of chromaticity would be required to provide sufficient Landau damping to overcome the instability.

These simulations can therefore be benchmarked during APEX studies by determining the chromaticity at which the beam becomes unstable with a bunch intensity of $1.5 \cdot 10^{11}$ protons per bunch. Furthermore, it is planned to determine the transverse impedance of RHIC by measuring bunch-by-bunch beam transfer functions (BTFs) for bunches of different intensities. 
Even below the instability threshold, electron cloud induced dynamic pressure rise has been observed to cause emittance growth [11]. However, the instability threshold has slowly increased over the years, from $1.3 \cdot 10^{11}$ protons per bunch in 2006 to beyond $2 \cdot 10^{11}$ protons per bunch in 109 bunches in 2011. In-situ coating with copper and amorphous carbon, as it is currently under study, would increase this threshold to bunch intensities where it would not longer be a limitation. As part of the APEX study program, it is foreseen to study this effect in detail at high intensity.

\section{Cryogenic BPM Cables}

The cryogenic BPM cables in RHIC are specified for a maximum temperature of $123 \mathrm{C}$, beyond which the insulation starts to deteriorate. Preliminary estimates were performed to determine the RHIC operational limits, as shown in Figure 9. Assuming beam orbit offsets of $3.5 \mathrm{~cm}$, which corresponds to the beam pipe aperture in the RHIC arcs, the rms proton bunch length must not be shorter than $32 \mathrm{~cm}$ at a bunch intensity of $3 \cdot 10^{11}$ protons per bunch in 109 bunches. If the maximum orbit offset is limited to $1 \mathrm{~cm}$, bunches as short as $17 \mathrm{~cm}$ are permissible. This condition requires an orbit interlock system that aborts the beam as soon as orbit offsets exceeding the safe limit are detected. For even shorter bunches the BPM cables would have to be replaced, which would take about two summer shutdowns. In addition, the DX BPM attenuators are being replaced to account for the higher bunch intensities, and heat sinks may have to be installed at all other BPM attenuators.

To check the validity of these calculations, a few BPM cables will be equipped with thermocouplers. These will provide online temperature measurements at the cryogenic BPM cables, so cable heating as a function of beam intensity, bunch length, and orbit displacement can be studied in detail.

\section{Resistive Wall Heating}

Since the stainless steel beam pipes in RHIC are not copper coated, their resistivity leads to parasitic losses that increase the heat load on the helium refrigerator. Calculations (Figure 10) show that the parasitic losses per ring do not exceed the $2 \mathrm{~kW}$ cryo limit [12] at $3 \cdot 10^{11}$ protons per bunch in 109 bunches as long as the rms bunch length is greater than $20 \mathrm{~cm}$. Shorter bunches would require in-situ coating of the cryogenic beam pipes with copper or another low-resistance material.

Experimental tests of resistive wall heating can be carried out parasitically, for instance during the scrubbing effort, or in parallel to other dedicated high intensity studies with short bunches, for example when studying the effect of these beams on the cryogenic BPM cables.

\section{Summary}

We have examined the main RHIC subsystems that need to be thoroughly tested and potentially upgraded to allow the proton beam intensity to be increased to $3 \cdot 10^{11}$ protons per bunch in 109 bunches per beam. Before any beam experiments at these intensities can be performed, precautions such as closing Thompson Road have to be taken [13].

\section{References}

[1] http://www.cadops.bnl.gov/AGS/Accel/SND/OPM/Ch02/02-05-02.PDF

[2] K. Yip, unpublished 


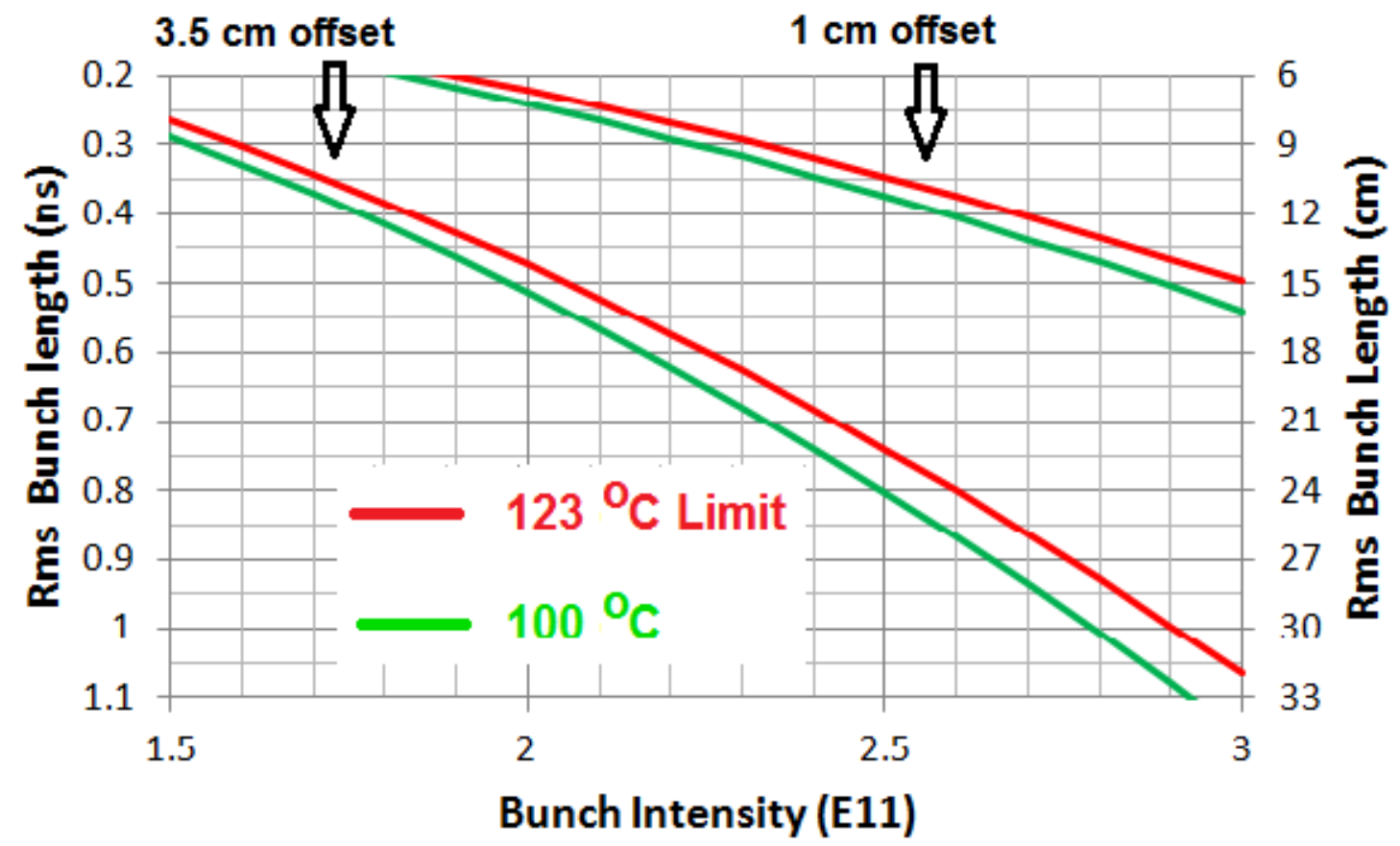

Figure 9: Preliminary estimates of RHIC operational limits due to cryogenic BPM cable heating. The specified maximum temperature allowed for these cables is 123 degrees C. Limiting the cable temperature to 100 degrees $\mathrm{C}$ provides some necessary safety margin. Cable frequency response measurements are underway over a wide temperature range and the results will be used to improve these estimates. It will also be determined what the real maximum operating temperature is for these cables since the values indicated above may be too conservative. 


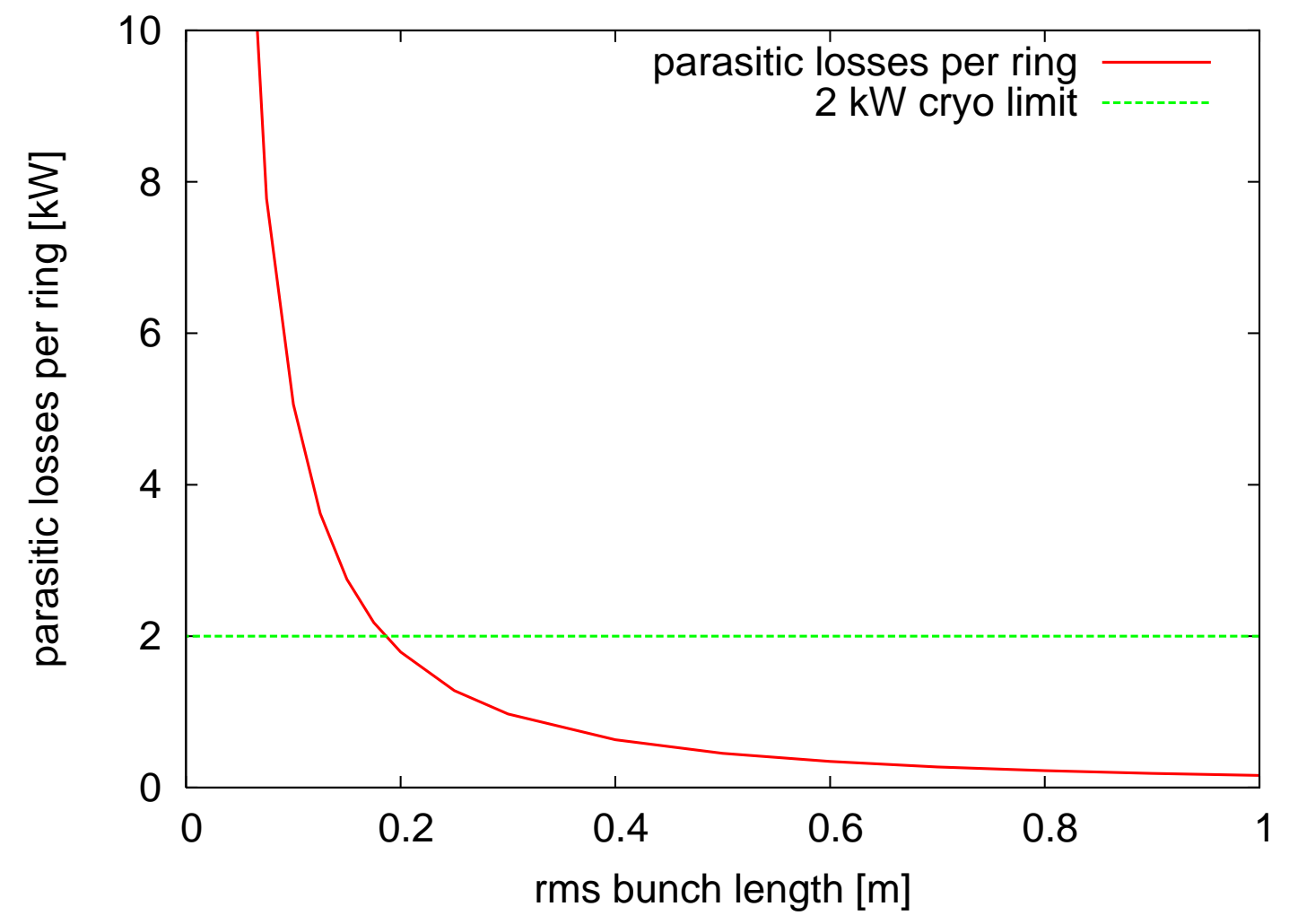

Figure 10: Parasitic losses due to resistive wall heating in one RHIC ring, together with the $2 \mathrm{~kW}$ cryo limit per ring. 
[3] S. Nayak, private communication

[4] R. Fliller, A. Drees, "Collimation", RHIC Retreat 2003, http://www.rhichome.bnl.gov/AP/RHIC2003/Retreat/

[5] G. Robert-Demolaize, "Simulations for a RHIC Collimation System Upgrade", RHIC Monday Meeting October 10, 2011, http://www.cadops.bnl.gov/AP/Meetings//RHIC_Monday_meetings/2011/October/

[6] J. M. Brennan, unpublished

[7] S. Y. Zhang et al., "Transverse impedance measurement at the RHIC", Proc. EPAC 2002

[8] R. Calaga, APEX Workshop 2010, unpublished

[9] M. Blaskiewicz, unpublished

[10] M. Blaskiewicz et al., "Longitudinal impedance measurement in RHIC", Proc. EPAC 2002

[11] S. Y. Zhang and V. Ptitsyn, Phys. Rev. ST Accel. Beams 11, 051001 (2008)

[12] Y. Than, private communication

[13] Minutes of Radiation Safety Committee of June 14, 2011 